\title{
Percutaneous Pulmonary Valve Implantation
}

\author{
Young-Seok Lee, MD'1 and Hyoung-Doo Lee, MD² \\ 1 Division of Pediatric Cardiology, Department of Pediatrics, Dong-A University Hospital, Busan, \\ ${ }^{2}$ Heart Center, Pusan National University Children's Hospital, Yangsan, Korea
}

\begin{abstract}
Pulmonary regurgitation (PR) is a frequent sequelae after repair of tetralogy of Fallot, pulmonary atresia, truncus arteriosus, Rastelli and Ross operation. Due to patient growth and conduit degeneration, these conduits have to be changed frequently due to regurgitation or stenosis. However, morbidity is significant in these repeated operations. To prolong conduit longevity, bare-metal stenting in the right ventricular outflow tract (RVOT) obstruction has been performed. Stenting the RVOT can reduce the right ventricular pressure and symptomatic improvement, but it causes PR with detrimental effects on the right ventricle function and risks of arrhythmia. Percutaneous pulmonary valve implantation has been shown to be a safe and effective treatment for patients with pulmonary valve insufficiency, or stenotic RVOTs.
\end{abstract}

(Korean Circ J 2012;42:652-656)

KEY WORDS: Catheterization; Pulmonary valve.

\section{Introduction}

Chronic pulmonary regurgitation (PR) is frequent sequelae after repair of tetralogy of Fallot. Chronic volume overload of the right ventricle, due to $P R$, causes ventricular dilatation and impairment of systolic and diastolic function. In the long term follow-up, PR causes atrial and ventricular arrhythmia, heart failure, and increased the risk of sudden death. An implantation of the right ventricle to pulmonary artery (PA) conduit is currently a well-established method of repair of various right ventricular outflow tract (RVOT) pathologies, including tetralogy of Fallot, pulmonary atresia and others. However, conduits have some limitations, as well. The inevitable degenerative changes of conduit lead to RVOT obstruction, PR and progressive conduit stenosis, due to somatic growth. It requires conduit replacement 10-15 years after the operation.

Since Bonhoeffer et al. ${ }^{1-3)}$ reported the first percutaneous pulmonary valve implantation in humans, many patients received this pro-

Correspondence: Young-Seok Lee, MD, Division of Pediatric Cardiology, Department of Pediatrics, Dong-A University Hospital, 26 Daesingongwon-ro, Seo-gu, Busan 602-715, Korea

Tel: 82-51-240-2634, Fax: 82-51-242-2765

E-mail: lyspedia@dau.ac.kr

- The authors have no financial conflicts of interest.

This is an Open Access article distributed under the terms of the Creative Commons Attribution Non-Commercial License (http://creativecommons. org/licenses/by-nc/3.0) which permits unrestricted non-commercial use, distribution, and reproduction in any medium, provided the original work is properly cited. cedure and has been an attractive alternative method of conduit replacement. This valve is made of bovine jugular vein sewn within a balloon expandable stent (Cheatham-Platinum stent) and handcrimped on a balloon in balloon catheter (Fig. 1). There is growing evidence of efficacy and safety for treatment of various congenital heart diseases, including PR and RVOT stenosis.

The Melody transcatheter pulmonary valve (Medtronic) received US Food and Drug Administration approval for human device exemption use in January 2010 and the Edwards SAPIEN transcatheter heart valve is still in clinical trials in the United States. ${ }^{4-6)}$ The SAPIEN transcatheter heart valve is larger in size than Melody valve. Others, like the Shelhigh injectable stented pulmonic valve and $\mathrm{Ed}$ wards-Cribier percutaneous heart valve is clinically undergoing investigation. ${ }^{78)}$ This article reviews the benefits and risks of percutaneous pulmonary valve implantation.

\section{Benefits of Percutaneous Pulmonary Valve Implantation}

Despite numerous studies of clinical outcomes in patients with postoperative RVOT dysfunction, the optimal timing of pulmonary valve replacement (PVR) and appropriate methods of treatment before irreversible dysfunction of right ventricle has not been determined. Clinical symptoms, including exercise intolerance, ventricular arrhythmia, and echocardiographic findings, such as progressive right ventricular (RV) dilatation and dysfunction, have been used as indications for operation. But there are concerns regarding the time 

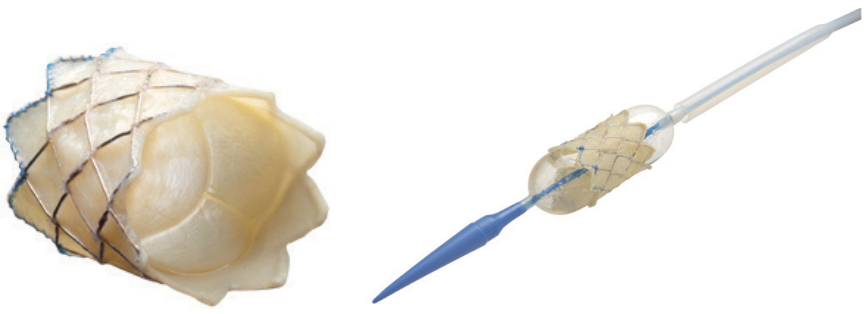

Fig. 1. Melody ${ }^{\circledR}$ transcatheter pulmonary valve and Ensemble ${ }^{\circledR}$ valve delivery system.

being too late for restoration of the RV function. Therrien et al.9) reported that post-operative RV remodeling, after PVR, may not occur in patients with RV end-diastolic volume $>170 \mathrm{~mL} / \mathrm{m}^{2}$ or endsystolic volume $>85 \mathrm{~mL} / \mathrm{m}^{2}$. Early PVR is safe and may prevent the detrimental complications of severe PR and improve long-term prognosis of the patients with PR. Although there is clear evidence that PR and RV pressure overload are deleterious, the incremental effects of progressive and increasingly chronic RV volume and pressure overload may be difficult to evaluate. Therefore, some investigators recommended early PVR surgery for PR, even absence of RV dilatation or dysfunction. Surgical conduit or PVR is the established standard treatment of care; nonetheless, patients with PR or RVOT conduit stenosis are often managed medically for many years before referral for surgery. The bovine jugular venous valved conduits have been used for RVOT reconstruction, but life span of the prosthetic conduits is limited. Calcification, stenosis, intimal proliferation, and graft degeneration causes progressive stenosis or regurgitation. Further, there still exists the morbidity of reoperations and the need to replace these valves surgically, particularly, when they are implanted at a young age.

The availability of less invasive options for PVR, such as transcatheter valve implantation, may provide a means of limiting the duration and severity of $\mathrm{RV}$ volume and/or pressure overload without increasing the lifetime number of open heart operations. The transcatheter intervention for PVR is less invasive, low risk of bleeding and infection, as well as low costs compared with surgical procedures. Also, there is significant improvement in exercise capacity and gas exchange efficiency during exercise after transcatheter valve implantation. ${ }^{101111}$

\section{Indications and Contraindications}

There are many indications by authors; recent indications were summarized in Table 1. ${ }^{1217128)}$ The patient selection for percutaneous transcatheter pulmonary valve implantation is crucial to the success. The most common cause of PR during late follow-up is tetralogy of Fallot and its variants. When associated with pulmonary atresia, a valved conduit is used for RVOT reconstruction. During late follow-
Table 1. Indications for percutaneous pulmonary valve implantation ${ }^{1217) 28 \text { ) }}$

Age $>5$ years, weight $>20 \mathrm{~kg}$

Conduit RVOT diameter $16-18 \mathrm{~mm} \leq 22 \mathrm{~mm}$

Severe PR, progressive RV dilatation and reduced RV function

Symptomatic patients with declining exercise tolerance ( $<65 \%$ of normals)

$R V$ systolic pressure $>2 / 3$ systemic pressure

Combination of stenosis and regurgitation with RV dysfunction and dilatation

Supraventricular or ventricular rhythm disturbances

RVOT: right ventricular outflow tract obstruction, PR: pulmonary regurgitation, RV: right ventricle

up, RVOT obstruction may develop for many kind of reason. For relief of RV pressure overload, due to stenosis, balloon dilatation and stent implantation have been successfully used in the conduits. ${ }^{13)}$ However, stent implantation alone cannot relieve PR and it may result in the exacerbation of the RV dysfunction. The limiting factor for valve implantation is the nature of RVOT and the size of conduit. Khambadkone and Bonhoeffer ${ }^{14)}$ reported that in patients with tetralogy of Fallot, native outflow tract with repair of only valvotomy or valvectomy may not provide secure landing zone. In case of outflow tract patch or transannular patch, the outflow tract tends to be dilated and may not have any secure implantation point. A large conduit, which is larger than the fully expanded dimension of the valve, is not good for device implantation. If the RVOT diameter is more than $22 \mathrm{~mm}$, the implanting zone is unlikely to hold a stent. Small sized conduit, especially below $16 \mathrm{~mm}$, which cannot be dilated to the diameter of the device. So, assessing the RVOT diameter is very important.

Khambadkone ${ }^{15)}$ summarized the indications as follows, 1) symptomatic patients with severe PR with RV dysfunction and/or dilatation, 2) patients with symptomatic arrhythmias and severe PR with RV dysfunction/dilatation, 3) severe PR and evidence of RV dysfunction and objective evidence of decreased exercise tolerance in asymptomatic patients, 4) patients with moderate or severe PR and additional lesions (residual ventricular septal defect, branch PA stenosis and tricuspid regurgitation) needing intervention, with or without symptoms, 5) asymptomatic patients with severe RVOT obstruction (RV pressures equal to or more than three-fourth systemic pressure) with or without PR, and 6) symptomatic patient with RVOT obstruction (RV pressure more than half of the systemic pressure) with or without PR.

Active infection is an absolute contraindication for this procedure. Care should be taken to get the previous history of conduit endocarditis, because endocarditis, within the conduit, can be underdiagnosed and may remain dormant, despite negative blood cultures and inflammatory markers. Because coronary artery abnormalities are not uncommon, computerized tomography (CT) angiography or 
Table 2. Summary of recent percutaneous pulmonic valve implantation

\begin{tabular}{|c|c|c|c|c|c|c|}
\hline & Patient no. & Age (years) & Weight (kg) & FU (Mo) & $\begin{array}{l}\text { Major complications } \\
\text { (immediate) }\end{array}$ & $\begin{array}{l}\text { Fracture } \\
\text { (\%) }\end{array}$ \\
\hline Khambadkone et al. ${ }^{29)}$ & 58 & 16 & 56 & 9.8 & $\begin{array}{l}2 \text { stent migration } \\
1 \text { homograft dissection }\end{array}$ & 12 \\
\hline Lurz et al. ${ }^{26)}$ & 155 & 21.2 & NA & 28.4 & $\begin{array}{l}5 \text { endocarditis } \\
2 \text { migration } \\
3 \text { homograft rupture } \\
1 \text { coronary compression }\end{array}$ & 21 \\
\hline Vezmar et al. ${ }^{28)}$ & 28 & 14.9 & 57.7 & 27.6 & $\begin{array}{l}1 \text { balloon rupture } \\
2 \text { conduit disruption }\end{array}$ & 10.7 \\
\hline McElhinney et $\left.a\right|_{.} ^{23)}$ & 136 & 19 & NA & NA & $\begin{array}{l}1 \text { coronary dissection } \\
1 \text { conduit rupture }\end{array}$ & 18 \\
\hline Zahn et al. ${ }^{17)}$ & 34 & 19.4 & NA & NA & $\begin{array}{l}1 \text { conduit rupture } \\
1 \text { PA perforation }\end{array}$ & 28 \\
\hline Eicken et al. ${ }^{12)}$ & 102 & 21.5 & 63 & 12 & 1 coronary compression & 5 \\
\hline Nordmeyer et al. ${ }^{16)}$ & 123 & 17.9 & NA & 13 & & 21.1 \\
\hline
\end{tabular}

Age, weight are expressed as median values and follow-up duration is expressed as mean value. FU: follow-up, Mo: months, PA: pulmonary artery, NA: not available

MRI should be done before catheterization. Patients less than $20 \mathrm{~kg}$ pose higher risk of complications and pregnancy is also contraindication.

\section{Complications}

The most frequent complications are stent migration and fracture. The conduits sometimes lie behind the sternum and this lead to external compression by the sternum and adhesions to the anterior chest wall. The stent fractures occur frequently, and require repeated balloon dilatation, another stent implantation or operation (Table 2). Peng et al. ${ }^{13)}$ reported stent fracture occurred in $43 \%$ of the patients who underwent cardiac catheterization before a conduit replacement surgery. The risk factors were external compression and substernal conduit location. Nordmeyer et al. ${ }^{16)}$ reported that stent fractures occur in about 20\% at the 2 year follow up, and substantial number of stent fractures occurred during the first 180 days. Associated risk factors for stent fractures were implantation into native RVOT, absence of calcification along the RVOT and greater recoil of valve after implantation. Zahn et al. ${ }^{17)}$ reported stent fracture rate of 28\% during the 6 months follow-up, and it resulted from excessive external loading forces. Restenosis of the valve may result from the stent fracture and it can be evaluated by $\mathrm{X}$-rays, echocardiography or CT. To reduce the stent fracture, presenting the conduit with bare-metal stents before implanting the valve is now widely accepted. ${ }^{18-21)}$ The principle of presenting is to reduce the compressive stress on the valve and preparing the landing zone of the valve with strong metal stents. It is also helpful in successfully relieved stenosis, particularly, where significant recoil is seen after predilation.
In patients with conduit stenosis, predilation with high pressure balloon can be helpful because it allows for easier implantation of the stent through large sheath. ${ }^{15)}$

Valve migration is a fearful complication, as well. Predictors of percutaneous pulmonary valve migration or embolization have never been clearly reported. Some expert opinion suggested that valvesize mismatch, difficulty with catheter manipulation, suboptimal implant location and RVOT hypermobility may be great cause of stent migration or embolization. ${ }^{22)}$ The management of percutaneous valve migration is variable and dependent on the size and location of the valve. If the valve migrates distally, it can be solved by expanding the valve within the branch PA. However proximal migration to the right ventricle requires surgical approach, because reposition of the valve back into the inferior vena cava (IVC) is usually not possible, due to large valve size and small IVC diameter.

After percutaneous valve implantation, stent embolization occurred not uncommonly. It occurs immediately after the procedure, some cases occur after a few months later. Meticulous follow-up is important and careful monitoring of sudden symptom of chest discomfort or arrhythmia is required.

And the most serious complication is coronary artery compression with stent and/or valve implantation. It is essential to assess the risk of coronary artery compression when placing a RVOT stent or percutaneous valve implantation. In case of RVOT reconstruction, such as tetralogy of Fallot, coronary arterial anatomy may be anomalous and care should be taken during the procedure. ${ }^{23)}$ If the RVOT conduit passes directly over a major coronary branch, the coronary artery can be compressed because of rigid stent expansion. Sridharan et al. ${ }^{24)}$ and Biermann et al. ${ }^{25)}$ reported a significant coronary ar- 
tery compression, which was demonstrated by balloon inflation within homograft to maximum diameter and simultaneous selective coronary angiography. If there are suspicions of coronary artery compression, selective coronary angiography with simultaneous balIon inflation in the RVOT is the key procedure to assess the spatial relationship between the coronary artery and stent.

Endocarditis is a fearful complication, as well; aseptic environment is important and treat patients with appropriate intravenous antibiotics. Other complications are bleeding, hematomas, fever, infection, right bundle branch block, and etc.

\section{Medium-Term Follow-Up Results}

After valve implantation, the RV systolic pressure and outflow gradient fall there is a significant improvement in PR. The results of the most recent large studies are listed in Table 1. During the follow up, there is early device failure (Hammock effect, stent fracture, residual stenosis), which leads to recurrent RVOT dysfunction. It is clinically important to know the mechanism of RVOT dysfunction and to know the treatment method to restore RV dysfunction. Lurz et al. ${ }^{26)}$ reported about learning curve in the procedure. They compare the results of pulmonary valve implantation between the early and late period that freedom form reoperation was significantly longer in the later period. They discussed about some factors regarding the contribution factors. One is reduced incidence of residual gradients. A postprocedural gradient $>25 \mathrm{~mm} \mathrm{Hg}$ after the procedure was risk factor for reoperation. So, they dilate with balloon aggressively after the valve implantation. The other factor is patient selection criteria, which is stricter than the early period. They consider careful evaluation of conduit type, RVOT morphology, and distensibility, which are very important in patient selection. As such, they do not attempt in patients with patch reconstruction of the RVOT and very distensible homograft. Nordmeyer et al. ${ }^{27)}$ reported that in cases of stent fracture, residual stenosis, repeat valve implantation is an effective treatment method for device failure and lengthen the freedom from reintervention. McElhinney et al. ${ }^{30)}$ reported recent results of US Melody valve trial that implant within an existing stent, new prestent, or bioprosthetic valve was associated with longer freedom from stent fracture. And valve compression and apposition to anterior chest wall were associated with short freedom from stent fracture.

Medium-term follow-up is good, and regurgitation due to degeneration of valve leaflet is very rare. Calcification of valve leaflets during the late follow-up is also rare. In late explants, there is very little endothelialization of the valve inside the stent. Freedom from reoperation was $70 \%$ at 5 years and freedom from transcatheter reintervention was $73 \%$ at 5 years. Survival at 83 months is $96.6 \%{ }^{26)}$
In terms of valve failure, more data is needed because follow up period is a little short.

\section{Summary}

Percutaneous pulmonary valve implantation is safe and effective method of treatment in patients with repaired congenital heart disease. Medium follow-up data shows good freedom from reoperation and valve implantation can delay open heart surgery. But care should be taken before procedure to evaluate possible coronary compression by stent or percutaneous valve implantation. Stent fractures were not uncommon after valve implantation and more likely in patients with severely obstructed conduits and located directly behind the anterior chest wall. However, it can be managed by reintervention with second stent. More careful consideration of RVOT morphology and prestent with bare metal stent maybe a good procedure before transcatheter pulmonic valve implantation. Longterm follow-up study is needed to evaluate valve function.

\section{References}

1. Bonhoeffer $P$, Boudjemline $Y$, Saliba Z, et al. Percutaneous replacement of pulmonary valve in a right-ventricle to pulmonary-artery prosthetic conduit with valve dysfunction. Lancet 2000;356:1403-5.

2. Bonhoeffer $P$, Boudjemline $Y$, Saliba $Z$, et al. Transcatheter implantation of a bovine valve in pulmonary position: a lamb study. Circulation 2000;102:813-6.

3. Bonhoeffer $P$, Boudjemline $Y$, Qureshi $S A$, et al. Percutaneous insertion of the pulmonary valve. J Am Coll Cardiol 2002;39:1664-9.

4. Lauten $A$, Hoyme $M$, Figulla HR. Severe pulmonary regurgitation after tetralogy-of-Fallot repair: transcatheter treatment with the Edwards SAPIEN XT heart valve. Heart 2012;98:623-4.

5. Kenny D, Hijazi ZM, Kar S, et al. Percutaneous implantation of the Edwards SAPIEN transcatheter heart valve for conduit failure in the pulmonary position: early phase 1 results from an international multicenter clinical trial. J Am Coll Cardiol 2011;58:2248-56.

6. Boone RH, Webb JG, Horlick $E_{1}$ et al. Transcatheter pulmonary valve implantation using the Edwards SAPIEN transcatheter heart valve. Catheter Cardiovasc Interv 2010;75:286-94.

7. Marianeschi SM, Santoro F, Ribera E, et al. Pulmonary valve implantation with the new Shelhigh Injectable Stented Pulmonic Valve. Ann Thorac Surg 2008;86:1466-71; discussion 1472.

8. Garay F, Webb J, Hijazi ZM. Percutaneous replacement of pulmonary valve using the Edwards-Cribier percutaneous heart valve: first report in a human patient. Catheter Cardiovasc Interv 2006;67:659-62.

9. Therrien J, Provost Y, Merchant N, Williams W, Colman J, Webb G. Optimal timing for pulmonary valve replacement in adults after tetralogy of Fallot repair. Am J Cardiol 2005;95:779-82.

10. Batra AS, McElhinney DB, Wang $W$, et al. Cardiopulmonary exercise function among patients undergoing transcatheter pulmonary valve implantation in the US Melody valve investigational trial. Am Heart J 
2012;163:280-7

11. Lurz P, Nordmeyer J, Giardini A, et al. Early versus late functional outcome after successful percutaneous pulmonary valve implantation: are the acute effects of altered right ventricular loading all we can expect? J Am Coll Cardiol 2011;57:724-31.

12. Eicken $A$, Ewert $P$, Hager $A$, et al. Percutaneous pulmonary valve implantation: two-centre experience with more than 100 patients. Eur Heart J 2011;32:1260-5.

13. Peng LF, McElhinney DB, Nugent AW, et al. Endovascular stenting of obstructed right ventricle-to-pulmonary artery conduits: a 15-year experience. Circulation 2006;113:2598-605.

14. Khambadkone S, Bonhoeffer P. Nonsurgical pulmonary valve replacement: why, when, and how? Catheter Cardiovasc Interv 2004;62:401-8.

15. Khambadkone S. Percutaneous pulmonary valve implantation. Ann Pediatr Cardiol 2012:5:53-60.

16. Nordmeyer J, Khambadkone $S$, Coats $L$, et al. Risk stratification, systematic classification, and anticipatory management strategies for stent fracture after percutaneous pulmonary valve implantation. Circulation 2007;115:1392-7.

17. Zahn EM, Hellenbrand WE, Lock JE, McElhinney DB. Implantation of the melody transcatheter pulmonary valve in patients with a dysfunctional right ventricular outflow tract conduit early results from the u.s. Clinical trial. J Am Coll Cardio/ 2009;54:1722-9.

18. Demkow M, Biernacka EK, Spiewak $M$, et al. Percutaneous pulmonary valve implantation preceded by routine prestenting with a bare metal stent. Catheter Cardiovasc Interv 2011;77:381-9.

19. Nordmeyer J, Lurz $P$, Khambadkone $S$, et al. Pre-stenting with a bare metal stent before percutaneous pulmonary valve implantation: acute and 1-year outcomes. Heart 2011;97:118-23.

20. Momenah TS, El Oakley R, Al Najashi K, Khoshhal S, Al Qethamy H, Bonhoeffer P. Extended application of percutaneous pulmonary valve implantation. J Am Coll Cardio/ 2009;53:1859-63.

21. Lurz $P$, Nordmeyer J, Muthurangu V, et al. Comparison of bare metal stenting and percutaneous pulmonary valve implantation for treatment of right ventricular outflow tract obstruction: use of an x-rayl magnetic resonance hybrid laboratory for acute physiological assessment. Circulation 2009;119:2995-3001.

22. Cubeddu RJ, Hijazi ZM. Bailout perventricular pulmonary valve implantation following failed percutaneous attempt using the Edwards Sapien transcatheter heart valve. Catheter Cardiovasc Interv 2011;77: 276-80.

23. McElhinney DB, Hellenbrand WE, Zahn EM, et al. Short- and mediumterm outcomes after transcatheter pulmonary valve placement in the expanded multicenter US melody valve trial. Circulation 2010;122: 507-16.

24. Sridharan S, Coats L, Khambadkone S, Taylor AM, Bonhoeffer P. Images in cardiovascular medicine. Transcatheter right ventricular outflow tract intervention: the risk to the coronary circulation. Circulation 2006: 113:e934-5.

25. Biermann D, Schönebeck J, Rebel M, Weil J, Dodge-Khatami A. Left coronary artery occlusion after percutaneous pulmonary valve implantation. Ann Thorac Surg 2012;94:e7-9.

26. Lurz $P$, Coats $L$, Khambadkone $S$, et al. Percutaneous pulmonary valve implantation: impact of evolving technology and learning curve on clinical outcome. Circulation 2008;117:1964-72.

27. Nordmeyer J, Coats L, Lurz P, et al. Percutaneous pulmonary valve-invalve implantation: a successful treatment concept for early device failure. Eur Heart J 2008;29:810-5.

28. Vezmar M, Chaturvedi R, Lee KJ, et al. Percutaneous pulmonary valve implantation in the young 2-year follow-up. JACC Cardiovasc Interv 2010;3:439-48.

29. Khambadkone $S$, Coats $L$, Taylor $A$, et al. Percutaneous pulmonary valve implantation in humans: results in 59 consecutive patients. Circulation 2005;112:1189-97.

30. McElhinney DB, Cheatham JP, Jones TK, et al. Stent fracture, valve dysfunction, and right ventricular outflow tract reintervention after transcatheter pulmonary valve implantation: patient-related and procedural risk factors in the US Melody Valve Trial. Circ Cardiovasc Interv 2011;4:602-14. 